\title{
Dependence and Fundamentality
}

\author{
Justin Zylstra \\ Department of Philosophy, University of Alberta
}

\begin{abstract}
I argue that dependence is neither necessary nor sufficient for relative fundamentality. I then introduce the notion of 'likeness in nature' and provide an account of relative fundamentality in terms of it and the notion of dependence. Finally, I discuss some puzzles that arise in Aristotle's Categories, to which the theory developed is applied.
\end{abstract}

Keywords: dependence, fundamentality, Aristotelian metaphysics

\section{Introduction}

The notion of dependence is an integral part of the neo-Aristotelian's armamentarium. For the task of metaphysics, on this conception, is to determine what is fundamental, and it is through the relation of dependence that what is fundamental is at least partially determined. It is thus part of the neoAristotelian view that dependence and fundamentality are intimately connected. ${ }^{1}$

But what, precisely, is the nature of this connection? For many, the two notions appear to go hand in hand, if it is not assumed that they are synonymous. Consider, for instance, the following snippets from the literature.

Ross Cameron $(2008,4)$ takes as primitive the notion of 'dependence', and defines from this a notion of 'independence', which he takes to be synonymous with 'fundamental': "An entity $x$ is ontologically independent (or fundamental) iff there is no entity $y$ such that $x$ ontologically depends on $y$, [and $x$ is] ontologically dependent otherwise".

Corresponding author's address: Justin Zylstra, Department of Philosophy, AH 2-25, University of Alberta, Edmonton, AB, T6G 2E7, Canada. Email: jzylstra@ualberta.ca.

1 On neo-Aristotelianism in contemporary metaphysics, see (Schaffer 2009, 351), where, for instance, it is claimed that, on the (neo-)Aristotelian conception, the task of metaphysics is to say what depends on what, and its method is to deploy diagnostics for what is fundamental, together with diagnostics for dependence. See also (Tahko 2012). 
Jonathan Schaffer $(2009,373)$ takes as primitive the notion of 'ground', which he takes to be synonymous with 'dependence', and defines from this the notion of 'fundamentality": "[T]he key notions of a fundamental entity (a prior, primary, independent, ground entity) and derivative entity (a posterior, secondary, dependent, grounded entity) can both be defined in terms of grounding (ontological dependence, priority in nature), as follows: ...x is fundamental $=_{d f}$ nothing grounds $x \ldots$ [and] $x$ is derivative $=_{d f}$ something grounds $x$ ".

Karen Bennett (2011b, 93) defines a 'building relation' as an asymmetric and irreflexive relation such that the 'input' relatum is more fundamental than the 'output' relatum and overlaps it. In response to her use of 'fundamental' in the definition of a building relation, she claims that "in the sense at hand, 'fundamental' appears to amount to 'not itself built', and ' $x$ is more fundamental than $y$ ' appears to amount to little more than ' $x$ at least partially builds $y$, but $y$ does not even partially build $x$ "'. She then continues in a footnote that " $[t]$ his is certainly a-if not the-central notion of fundamentality in the literature. (For one example, note how Schaffer defines fundamentality as ungroundedness in 2009, Section 3.1.)"

Bennett's notion of a building relation is perhaps more general than the notion of dependence with which Cameron and Schaffer are concerned, and with which we will be concerned here. In any case, her claim about the literature suggests a standard of equivalence connecting dependence (or, perhaps, whatever metaphysical structuring relation you like) and fundamentality, and this standard is upheld by both Cameron and Schaffer with respect to dependence.

Granted, the above considerations are not decisive in establishing equivalence as the standard view about the connection between dependence and fundamentality. In any case, it is a good starting point for inquiry. For it is at the very least taken for granted by some, and, for those who may deny it, no systematic reason for its failure has been provided, nor is there any positive account of fundamentality on offer with which to replace it. ${ }^{3}$

2 Although Schaffer uses the term 'ground', he is just as well seen as giving an account of dependence. Both Cameron and Schaffer take independence to go hand in hand with absolute fundamentality. But given the existence of chains of dependence, the view that dependence and relative fundamentality go hand in hand is a natural extrapolation. Also, both Cameron and Schaffer take dependence as primitive, and not contained within the essentialist framework, as it is for others (e.g. Fine 1995b), and as it will be here. But this is beside the point of how they connect dependence and fundamentality.

3 We separate grounding (e.g. Fine 2012) from dependence (e.g. Fine 1995b), though we, at least for heuristic purposes, may allow one to play a role in the articulation of the other. But it is worth noting that, on the topic of grounding, there is dissent from equivalence. First, Kit Fine (2009) denies that grounding is sufficient for relative fundamentality. On his view, 
So let us take equivalence as our starting point. Letting ' $x>y$ ' abbreviate ' $x$ depends on $y$ ' and ' $f(y, x)$ ' abbreviate ' $y$ is more fundamental than $x$ ', we state the view as follows.

(EQU) $\forall x \forall y(x>y \equiv f(y, x)) .{ }^{4}$

(Dependence is equivalent to relative fundamentality.)

From (EQU) we may infer the following two theses.

(SUF) $\forall x \forall y(x>y \supset f(y, x))$. (Dependence is sufficient for relative fundamentality.)

(NEC) $\forall x \forall y(f(y, x) \supset x>y)$.

(Dependence is necessary for relative fundamentality.)

In this paper I argue that (EQU) is false in both directions: that both (SUF) and (NEC) are false. Although I treat the arguments against each separately, both take their cue from this passage in Aristotle's Categories.

Substance, it seems, does not admit of a more and a less. I do not mean that one substance is not more a substance than another (we have said that it is) but that any given substance is not called more, or less, that which it is. For example, if this substance is a human being, it will not be more a human being or less a human being than itself or another human being. (3 $\mathrm{b}_{32}-4 \mathrm{a} 9$, Ackrill transl., slightly modified).

The claim here is not, as Aristotle says, that no substance is more fundamental, or more a substance, than any other substance. For he claims that primary substances, such as Socrates, are more fundamental than secondary substances, such as the species human being; and he claims that specific substances, such as human being, are more fundamental than generic

an additional bit of ideology is required, namely a 'reality' operator. But I am inclined to think that most grounding theorists do not take on this bit of extra ideology. Second, Karen Bennett (2011a) denies that grounding is necessary for relative fundamentality, although she affirms that it is sufficient (perhaps with the additional claim that ungrounded truths actually ground something). Third, Kathrin Koslicki (forthcoming) rejects equivalence for grounding, but because she rejects grounding altogether as a theory of fundamentality.

Dissent from equivalence has been less vocalized with respect to dependence. It is perhaps only Koslicki (2013) who suggests that dependence is not sufficient for relative fundamentality, though allows that it may be necessary. Interestingly, Koslicki (forthcoming) develops a 'multi-dimensional' view of fundamentality in response to her reaction against grounding, a view which involves the notion of dependence. But some dimensions of her multi-dimensional view generate the same problems we will raise here against equivalence. So her dissent from the standard, and the positive view she puts in its place, are not immune to the contents of this paper.

${ }^{4}$ Although we omit necessity operators, we commit ourselves to the stronger theses involving boxes before and after the quantifers. 
substances, such as animal. The claim is rather that, for any items which are essentially alike, or alike in nature (i.e. for any items that share in the what-it-is), they do not differ with respect to fundamentality.

We shall understand the claim that no two items which are alike in nature differ with respect to fundamentality as a conjunction of the following two claims, letting ' $n(x, y)$ ' abbreviate ' $x$ and $y$ are alike in nature'.

1. $\forall x \forall y(n(x, y) \supset \neg(f(y, x) \vee f(x, y)))$.

(For any pair of items alike in nature, neither is more fundamental than the other.)

2. $\forall x \forall y(n(x, y) \supset \forall z(f(y, z) \equiv f(x, z))) .^{5}$

(Any items alike in nature are not differently fundamental.)

Call the conjunction of (1) and (2) '(TNC)' for 'The Nature Constraint'. I argue that (SUF) and (NEC) fail on the basis of (TNC). The upshot is that (NEC) underpredicts relations of relative fundamentality (i.e. predicts that there is not relative fundamentality where there is), and (SUF) overpredicts relations of relative fundamentality (i.e. predicts that there is relative fundamentality where there is not). (TNC) brings out these deficiencies of (EQU).

After giving the arguments against (NEC) and (SUF), I develop a positive account of fundamentality, central to which are the notions of dependence and likeness in nature. The ideology of fundamentality is then seen to be derivative from that of dependence and likeness in nature, which are themselves derivative from the ideology of essence. So we shall arrive at a predictively successful theory of fundamentality that respects the neo-Aristotelian conception, and which does so without ideological superfluity. Finally, I discuss some puzzles that arise in Aristotle's Categories, to which the theory developed is applied.

\section{Dependence and Likeness in Nature}

The theses of (EQU) and (TNC) involve the notions of dependence and likeness in nature. In this section we specify how we understand these notions.

5 Strictly, we should think of (2) as being itself conjunctive, adding the claim that $\forall x \forall y(n(x, y) \supset \forall z(f(z, y) \equiv f(z, x)))$. For items alike in nature should neither differ with respect to what is more fundamental than them nor to what is less fundamental than them. The arguments advanced against (SUF) and (NEC) require only one conjunct.

Furthermore, given that relative fundamentality is irreflexive, i.e., $\forall x \neg f(x, x)$, (2) implies (1). For suppose (2) but not (1). It follows from the falsity of (1) that there is some $x$ and $y$ such that $n(x, y)$ and $f(y, x) \vee f(x, y)$. Suppose that $n(a, b)$ and that either $f(a, b)$ or $f(b, a)$. Suppose then that $f(a, b)$. Given irreflexivity, $\neg f(b, b)$, and so, contra (2), there is some $\mathrm{z}$ such that $f(a, z) \wedge \neg f(b, z)$, namely $b$. The argument is similar if we suppose $f(b, a)$. 


\subsection{Dependence}

The sort of dependence with which we are concerned is metaphysical in character. Its metaphysical character is the result of fixing the 'strength' of the relation. Statements of metaphysical dependence are given within the scope of an essential operator. ${ }^{6}$

Following Fine (1995a), we take it that, associated with each predicate $F$, there is a 'source sensitive', or essential, operator $\square_{F}$. The interpretation of $\square_{F} \phi$, for some statement $\phi$, is that it is true in virtue of the identities of the items satisfying $F$ that $\phi$. Following Fine (1995b), this is taken to express a primitive relation between the items satisfying $F$ and the proposition expressed by $\phi .^{7}$ For instance, where $F=\lambda x(x=$ Socrates $)$, and $\phi=$ Socrates is human, $\square_{\lambda x(x=\text { Socrates })}$ (Socrates is human) says that it is true in virtue of the identity of Socrates that Socrates is human. ${ }^{8}$ Statements of metaphysical dependence are then partly formed by a statement within the scope of an essential operator, the delimiter of which applies to the dependent item.

But metaphysical dependence comes in different 'flavors'. For no item will be said to depend unqualifiedly on some item, but rather to depend for its having this or that factual status on some item, by virtue of essentially standing in some relation to that item, if it has that factual status. For instance, the proposition $A \vee B$ may depend for its being on having both $A$ and $B$ as constituents, though it may depend for its truth on being grounded either in $A$ or in $B$.

So in addition to being within the scope of an essential operator, statements of metaphysical dependence are conditional: as part of the essence of the dependent item, its having some factual status is conditional on its bearing some relation to the items on which it depends.

We can then give the following general framework for metaphysical dependence, where $F$ is some factual status and $R$ is some relation.

(RMD) $\forall x \forall y\left(x>y \equiv \exists F \exists R \square_{x}(F x \supset R x y)\right)$.

${ }^{6}$ Perhaps there are legitimate modal notions of dependence, where the modality employed is metaphysical necessity. Such notions of dependence might even be deserving of the name 'metaphysical'. Be that as it may, our concern is with a stricter, essentialist notion.

7 The items to which the delimiter applies need not perfectly coincide with the subjects of $\phi$. For instance, although Socrates and \{Socrates\} are subjects of 'Socrates is an element of $\{$ Socrates\}, the proposition is true in virtue of the identity of \{Socrates\} taken on its own. Given the relational construal of statements of essence, and a general unconcern for ontological cost-savings here, I move freely between word and world talk.

8 Although the delimiter of the operator is formulated in terms of predicates, we shall often use proper names instead. So instead of saying it is true in virtue of the identity of $\lambda x(x=$ $y$ ) that ..., we shall say 'it is true in virtue of the identity of y that .... Moreover, although, strictly, we read the operator as 'it is true in virtue of the identity of $\mathrm{x}$ that ...', we shall often omit the italicized bit. Both are intended to ease expression. 
( $x$ metaphysically depends on $y$ if and only if there is some factual status $F$, and some relation $R$, such that it is true in virtue of $x$ that $F x$ only if $R x y$. $)^{9}$

But (RMD) gives us only a notion of rigid metaphysical dependence. We define generic dependence as follows.

(GMD) $\forall x \forall y\left(D x \equiv \exists F \exists R \square_{x}(F x \supset \exists y R x y)\right)$.

( $x$ is dependent if and only if there exists some factual status $F$, and there exists some relation $R$, such that it is true in virtue of $x$ that $F x$ only if there exists a $y$ such that $R x y$.)

The analysandum of (GMD) involves a monadic predicate, read as ' $\mathrm{x}$ is dependent', as opposed to a binary relation. The reason is that it would otherwise be difficult to determine exactly what the right-side relatum is supposed to be in any particular case of generic dependence..$^{10}$ Note that every item that rigidly metaphysically depends on some item is dependent, though not vice versa. ${ }^{11}$ We then obtain the various flavors of metaphysical dependence by restricting our factual status, to existence, being, truth, obtaining, or what have you. ${ }^{12}$

9 In addition, we place some formal constraints on $R$. We shall say that $R$ is a strict partial ordering relation (i.e. irreflexive, asymmetric, and transitive).

${ }^{10}$ See (Barnes 2012) for a similar sentiment.

${ }^{11}$ (EQU) gives rigidified conditions for relative fundamentality. But we might put forward the following generic version of (EQU): $\forall x \forall y((D x \wedge \exists \phi(\phi(y)) \wedge \phi<D x) \equiv f(y, x))$. We use ' $<$ ' to indicate a relation of strict partial ground, and ' $\phi(y)$ ' to indicate that $y$ is involved in $\phi$. Given the general presumption that existential truths are grounded in their instances, the idea is that any item involved in an instance that grounds the existential, $\exists y R x y$, is more fundamental than the dependent item $x$. This is what we take to be expressed by ' $\phi(y)<D x^{\prime}$, or that $\phi$, which involves $y$, partially grounds $x$ 's being dependent. On the notion of strict partial ground, see (Fine 2012)

Given that every item that rigidly depends on something is dependent, we can say, in general, that $f(y, x) \equiv \exists \phi(\phi(y) \wedge \phi<D x)$. Then, for instance, $f$ (Socrates, $\{$ Socrates $\})$, since Socrates is a member of \{Socrates\} involves Socrates and grounds $\exists x$ ( $x$ is a member of $\{$ Socrates $\}$ ), where $R=$ membership. In a similar fashion, Socrates is more fundamental than the property being pale, since Socrates instantiates the property being pale involves Socrates and grounds $\exists x$ ( $x$ instantiates the property being pale). However, in the latter, merely generic case, the property's being dependent admits of alternative grounds, say, in Plato's being pale. But until we develop a positive account of relative fundamentality in Section 4, we shall continue to work with the rigidified version of (EQU), as it will suffice for constructing the arguments.

${ }^{12}$ We distinguish being from existence. When the flavor of dependence is existential, we use an existence predicate $E$ as our factual status. When the flavor is ontological, we use the existential quantifier $\exists$ for being. One benefit of this distinction is that, given that the essential operator is closed under logical consequence, every item $x$ is such that it is true 
Our concern in this paper is with ontological dependence. So let us restrict the factual status to being. Thus we should hitherto read (EQU), (SUF), and (NEC), and the '>' therein, as involving the notion of ontological dependence. Ontological dependence is then (RMD) where $F=\exists \cdot{ }^{13}$

For instance, $\{$ Socrates\} ontologically depends on Socrates, since it is true in virtue of $\{$ Socrates $\}$ that $\exists x(x=\{$ Socrates $\})$ only if Socrates is an element of \{Socrates\}. Given the formal constraints we've placed on $R$, we take the transitive closure of membership as our relation. By way of further example, Socrates' paleness trope will ontologically depend on Socrates, since it is true in virtue of Socrates' paleness trope that $\exists x\left(x=\right.$ Socrates' $^{\prime}$ paleness trope) only if Socrates' paleness trope is particularized from Socrates. ${ }^{14}$

\subsection{Likeness in Nature}

Let us assume that the essence of an item is given by the set of truths in virtue of its identity, or, alternatively, by the properties it has in virtue of its identity. It is then natural to understand likeness in nature as overlap of essence. ${ }^{15}$

For instance, Plato and Aristotle are alike in nature, since they both have

in virtue of it (or of anything else) that $\exists y(x=y)$. But we should hardly think that every item essentially exists. Still less should we think that every item's existence is true in virtue of any item taken on its own.

${ }^{13}$ It seems that our notion of ontological dependence is in keeping with Aristotle. For instance, Corkum (2008) suggests a general framework for Aristotle in Categories, according to which $A$ is ontologically independent from $B$ just in case $A$ admits of the ontological status of a being independently of standing in some tie to $B$. Corkum goes on to say that $A$ is ontologically dependent on $B$ if $A$ has the ontological status of a being in virtue of standing in some tie to $B$ (Corkum 2008, 77). Taking 'in virtue of' as indicating some tie in virtue of the identity of the dependent item, and taking being as expressed by the existential quantifer, the two coincide.

${ }^{14}$ We use this notion of 'being particularized from' instead of the bearing relation. It may be that any trope depends, existentially, on being borne by its bearer; but the dependence is perhaps not ontological, insofar as (i) essential truths are metaphysically necessary; (ii) a trope is borne by its bearer if and only if the bearer has the property; and (iii) (necessarily) everything is necessarily such that there is something to which it is identical. Given that tropes are particularized properties, it seems to me that there is a form of ontological dependence of trope on bearer analogous to that of proposition on constituent.

${ }^{15}$ Kit Fine (1995a) distinguishes the sentential mode from the predicate mode of expressing statements of essence. In the sentential mode, we say that a proposition is true in virtue of an item, and the essence of that item is the collection of propositions that are true in virtue of it. In the predicate mode, we say that a property is had in virtue of an item, and the essence of an item is the collection of properties had in virtue of that item. Although Fine himself adopts the sentential mode, the predicate mode is better for expressing claims of likeness in nature, since the sentential mode will involve propositions tailored to the item(s) in virtue of which they're true. For instance, it is true in virtue of Socrates that Socrates is human, and true in virtue of Plato that Plato is human, though it is neither true in virtue of Socrates that Plato is human nor true in virtue of Plato that Socrates is human. 
the property being human in virtue of their identities. Moreover, Socrates and Seabiscuit are alike in nature, since they both have the property being an animal in virtue of their identities. However, given some formal and material considerations of the essential operator, likeness in nature cannot be given merely in terms of overlap of properties had in virtue of some items. Some constraints are in order.

First, Fine's source-sensitive operator has the formal property of being closed under logical consequence: anything that follows from a truth in virtue of some items is true in virtue of those items. For instance, given that it is true in virtue of Socrates that Socrates is human, and Socrates' being human or an electron follows from Socrates' being human, it is true in virtue of Socrates that Socrates is human or an electron. But given that it is true in virtue of some electron $e_{1}$ that it is an electron, closure implies also that it is true in virtue of $e_{1}$ that it is human or an electron.

But we do not want to say that Socrates and $e_{1}$ are alike in nature because they both have the property being human or an electron in virtue of what they are. So we restrict claims of likeness in nature to the non-consequential, 'constitutive' properties had by items in virtue of their identities. ${ }^{16}$

Second, it may seem that everything is alike in nature to some degree. For instance, Socrates and the number 2 are alike in nature to some degree, since they are both by nature entities. Moreover, $e_{1}$ and Socrates are alike in nature to some degree, since they are both by nature material objects. But then given (TNC), no entities, or no material objects, could differ with respect to relative fundamentality. The result would be a conception of reality on which reality is flat.

We should think of properties as being 'definable', in the Aristotelian sense of admitting of a real definition in terms of genera and differentiae, which we express by the locution 'to be $F$ is to be $\phi$ ', where $\phi$ is some complex expression with a genus-differentiae structure. For instance, to be human is to be a mortal rational animal, to borrow an example from Aristotle. ${ }^{17}$

We might then say that being an entity is indefinable, on the grounds that its complement is null, and so there is no higher genus in terms of which it may be defined. Perhaps it itself is not a genus for this reason. Then we may also take as indefinable any properties whose definitions are given in terms of properties with null complements, for instance, being a material object (or being concrete), if to be a material object (or to be concrete) is to be a

${ }^{16}$ On the distinction between constitutive and consequentialist essence, see (Fine 1995b).

${ }^{17}$ Call statements of the form 'to be $F$ is to be $\phi$ ' statements of 'generic essence'. For a defense of generic essence, see (Correia 2006). Correia does not commit himself to $\phi$ having a genus-differentiae structure. See also (Koslicki 2012, Section 7.4) for a good discussion of real definition. 
spatiotemporally extended entity. ${ }^{18}$

It might be objected that the definability constraint is ad hoc, serving only to preserve (TNC) in the interest of arguing against (EQU). I disagree. Insofar as some items $x$ and $y$ are alike in nature because what $x$ is and what $y$ is are the same, I am inclined to think that some items are, if you like, 'essentially unalike'. Moreover, I am inclined to think that, although some items might be more similar in nature to some items than to others (in virtue of being material and not, say, numbers or sets), the respect in which they're similar is not enough to constitute their being alike in nature. So I think that the definability constraint is independently motivated, albeit perhaps by some intuitive understanding of what it means to be alike in nature. ${ }^{19}$

We thus arrive at the following account of likeness in nature: $n(x, y)$ if and only if there exists an $F$ such that it is true in virtue of $x$ that $F x$ and it is true in virtue of $y$ that $F y$, and $F$ is constitutive and definable.

\section{Against (NEC)}

Recall that (NEC) says that if $y$ is more fundamental than $x$, then $x$ ontologically depends on $y$, in symbols, $f(y, x) \supset x>y$. On the basis of (TNC), we may develop the following argument against (NEC), using sets and urelements as our example.

Suppose that

$$
\{\text { Socrates }\}>\text { Socrates. }
$$

Let us suppose that (SUF) is true, since we have yet no reason to deny it. Then it follows from (SUF) by universal instantiation and modus ponens that

$$
f(\text { Socrates, }\{\text { Socrates }\}) .^{20}
$$

Moreover, given that

$$
\neg(\{\text { Socrates }\}>\text { Plato }),
$$

it follows from (NEC) by universal instantiation and modus tollens that

$$
\neg f(\text { Plato, }\{\text { Socrates }\}) \text {. }
$$

${ }^{18}$ If we consider the more general property being material, there would seem to be no property corresponding to a genus, whose complement is non-null, and within which the property being spatiotemporally extended differentiates a species.

${ }^{19}$ Similar remarks apply, I think, to the constitutive constraint.

${ }^{20}$ In principle, we do not need (SUF) if we take the judgment of relative fundamentality as a stand-alone assumption. 
However, by universal instantiation on (2), we get that if

$$
n(\text { Socrates, Plato })
$$

then

$$
\forall z(f(\text { Socrates }, z) \equiv f(\text { Plato }, z)) .
$$

We assert that

$$
n(\text { Socrates, Plato })
$$

since both are by nature human, and being human is constitutive and definable. So,

$$
\forall z(f(\text { Socrates }, z) \equiv f(\text { Plato }, z)) .
$$

But we've established from (NEC) that

$$
\exists z \neg(f(\text { Socrates, } z) \equiv f(\text { Plato }, z)) \text {, namely \{Socrates\}. }
$$

One might object that (2) is too strong. It is perhaps unclear why two items which are alike in nature cannot be more fundamental than different items (or have different items more fundamental than them). But I am inclined to think that such a claim is part of our 'global' claims of relative fundamentality, for instance, that urelements are more fundamental than sets, or that particulars are more fundamental than tropes (given a substance-attribute theory of particularity).

Ontological dependence and relative fundamentality then differ in this respect. In the case of ontological dependence, if, in general, we assert that items of type A ontologically depend on items of type B, this implies that, for every element $a$ of type A, there exists some item $b$ of type B such that $a$ ontologically depends on $b .^{21}$ By contrast, if, in general, we assert that items of type $B$ are more fundamental than items of type $A$, this implies that, for every element $b$ of type $\mathrm{B}$, and for every element $a$ of type A, $b$ is more fundamental than $a$.

${ }^{21}$ We take up the relative fundamentality of the null set in Section 4. But the claim about dependence can be made consistent with the nature of the null set if we think of the identity of sets as given by the results of certain inputs into the set-builder (i.e. if we think of the nature of sets as given 'operationally', as in (Fine 2010)). Then it is true in virtue of \{Socrates\} that it is the result of the input Socrates, whereas it is true in virtue of the null set that it is the result of the 'zero' input. So there is something-namely, an input-on which the null set depends, but it is the input consisting of zero objects. We should then understand our global claim of dependence as saying that sets (i.e. the results of the set builder) are dependent on their inputs. See (Fine 2012) on the analogous notion of being 'zero grounded'. 


\section{4. $\quad$ Against (SUF)}

Recall that (SUF) says that if $x$ ontologically depends on $y$ then $y$ is more fundamental than $x$, in symbols, $x>y \supset f(y, x)$. On the basis of (TNC), we may develop the following argument against (SUF). We repeat the argument using different cases.

\subsection{Sets}

Suppose that

$$
\{\{\text { Socrates }\}\}>\{\text { Socrates }\} .
$$

From this it follows by (SUF) that

$$
f(\{\text { Socrates }\},\{\{\text { Socrates }\}\}) .
$$

By universal instantiation on (1), it follows that

$$
\begin{aligned}
& n(\{\text { Socrates }\},\{\{\text { Socrates }\}\}) \supset \neg(f(\{\text { Socrates }\},\{\{\text { Socrates }\}\}) \vee \\
& f(\{\{\text { Socrates }\}\},\{\text { Socrates }\})) .
\end{aligned}
$$

We assert that

$$
n(\{\text { Socrates }\},\{\{\text { Socrates })\}\}),
$$

since both are by nature sets, and being a set is constitutive and definable. Therefore,

$$
\neg(f(\{\text { Socrates }\},\{\{\text { Socrates }\}\}) \vee f(\{\{\text { Socrates }\}\},\{\text { Socrates }\})),
$$

and so

$$
\neg f(\{\text { Socrates }\},\{\{\text { Socrates }\}\}){ }^{22}
$$

The above argument relied only on (1) from (TNC). But the result can be obtained on the basis of (2) as well, at least given the irreflexivity of relative fundamentality. For given that

$$
\{\{\text { Socrates }\}\}>\{\text { Socrates }\},
$$

(SUF) implies that

$$
f(\{\text { Socrates }\},\{\{\text { Socrates }\}\}) .
$$

${ }^{22}$ You might think that the rank of a set is a feature between which two sets can differ in nature subordinately to being a set. I disagree. I do not think that rank marks a difference in nature for sets any more than generation marks a difference in nature for, say, human beings. 
By universal instantiation on (2), it follows that

$$
\begin{aligned}
& n(\{\text { Socrates }\},\{\{\text { Socrates }\}\}) \supset \forall z(f(\{\text { Socrates }\}, z) \equiv \\
& f(\{\{\text { Socrates }\}\}, z)) .
\end{aligned}
$$

We assert that

$$
n(\{\text { Socrates }\},\{\{\text { Socrates }\}\}),
$$

since both are by nature sets, and being a set is constitutive and definable. Given our added premise, it follows that

$$
\neg f(\{\{\text { Socrates }\}\},\{\{\text { Socrates }\}\}) .
$$

We conclude from this then that

$$
\exists z \neg(f(\{\text { Socrates }\}, z) \equiv f(\{\{\text { Socrates }\}\}, z)) \text {, namely }\{\{\text { Socrates }\}\} .
$$

\subsection{Origins}

Suppose some version of the essentiality of origin thesis is true, and that I depend for what I am on my parents. Suppose that

$$
\text { me }>\text { mother. }
$$

Then (SUF) implies that

$$
f(\text { mother, me). }
$$

By universal instantiation on (1), it follows that

$$
n(\text { mother, me }) \supset \neg(f(\text { mother, me }) \vee f(\text { me, mother })) \text {. }
$$

We assert that

$$
n(\text { mother, me), }
$$

since we are both by nature human, and being human is constitutive and definable. Therefore,

$$
\neg(f(\text { mother, me }) \vee f(\text { me, mother })),
$$

and so

$$
\neg f(\text { mother, me }) \text {. }
$$

Alternatively, we may proceed by (2). By universal instantiation on (2), it follows that

$$
n(\text { mother, } m e) \supset \forall z(f(\text { mother, } z) \equiv f(\text { me }, z)) .
$$


We assert that

$$
n \text { (mother, me), }
$$

since we are both by nature human, and being human is constitutive and definable. However, given the irreflexivity of relative fundamentality,

$$
\neg f(m e, m e) .
$$

Therefore,

$$
\exists z \neg(f(\text { mother, } z) \equiv f(m e, z)), \text { namely me. }
$$

\subsection{Natural Numbers}

Suppose that every natural number with a predecessor depends for what it is on at least its immediate predecessor, given that it is by nature the successor of its immediate predecessor. Suppose, for instance, that

$$
2>1 .
$$

Then (SUF) implies that

$$
f(1,2) \text {. }
$$

By universal instantiation on (1), it follows that

$$
n(1,2) \supset \neg(f(1,2) \vee f(2,1)) .
$$

We assert that

$$
n(1,2) \text {, }
$$

since both are by nature natural numbers, and being a natural number is constitutive and definable. Therefore,

$$
\neg(f(1,2) \vee f(2,1)),
$$

and so

$$
\neg f(1,2) .
$$

Alternatively, we may proceed by (2). By universal instantiation on (2), it follows that

$$
n(1,2) \supset \forall z(f(1, z) \equiv f(2, z)) .
$$

We assert that

$$
n(1,2) \text {, }
$$


since we are both by nature natural numbers, and being a natural number is constitutive and definable. However, given the irreflexivity of relative fundamentality,

$$
\neg f(2,2) .
$$

Therefore,

$$
\exists z \neg(f(1, z) \equiv f(2, z)) \text {, namely } 2 .
$$

\subsection{Sums}

Suppose that sums depend for what they are on their mereological parts. Suppose, for instance, that

$$
a b c>a b,
$$

where $a b c$ is the sum of $a, b$, and $c$. Then (SUF) implies that

$$
f(a b, a b c) \text {. }
$$

By universal instantiation on (1),

$$
n(a b, a b c) \supset \neg(f(a b c, a b) \vee f(a b, a b c)) .
$$

We assert that

$$
n(a b, a b c),
$$

since both are by nature sums, and being a sum is constitutive and definable. Therefore,

$$
\neg(f(a b, a b c) \vee f(a b c, a b)),
$$

and so

$$
\neg f(a b, a b c) .
$$

Alternatively, we may proceed by (2). By universal instantiation on (2), it follows that

$$
n(a b, a b c) \supset \forall z(f(a b, z) \equiv f(a b c, z)) .
$$

We assert that

$$
n(a b, a b c),
$$

since both are by nature sums, and being a sum is constitutive and definable. However, given the irreflexivity of relative fundamentality, 
$\neg f(a b c, a b c)$.

Therefore,

$$
\exists z \neg(f(a b, z) \equiv f(a b c, z)) \text {, namely } a b c .
$$

The arguments against (SUF) can be derived on the basis of either (1) or (2). If one is convinced that (2) is true, then (1) is not required (though (2) implies (1)). But I am inclined to think that (1) is much less controversial than (2), although (SUF) is a much more plausible thesis than (NEC). That any items alike in nature are such that neither is more fundamental than the other is quite plausible. How, for instance, could one human being be more fundamental than another? That any items alike in nature are 'equivalently fundamental' is perhaps more controversial, but is, I think, in keeping with global assertions of relative fundamentality. So perhaps relying on the less controversial (1) bolsters the argument against (SUF), as one might be less inclined to give up on this direction of (EQU).

\section{Relative Fundamentality}

It has been argued that both (NEC) and (SUF) are false. But it remains to be specified exactly when relative fundamentality occurs. In this section we develop a positive account of relative fundamentality.

Our arguments against (EQU) were constructed using a rigidified version of (EQU). This was sufficient for giving the arguments of the previous sections. But ideally, a positive account of relative fundamentality should be built to handle both rigid and non-rigid cases. Recall the non-rigidified equivalence thesis, which states that $f(y, x) \equiv \exists \phi(\phi(y) \wedge \phi<D x)$, i.e., that $y$ is more fundamental than $x$ if and only if there is some truth that involves $y$ and partially grounds $x$ 's being dependent. The non-rigidified equivalence thesis is false, since the rigidified equivalence thesis is false. But we shall make use of this notion of a 'dependent', and the notion of 'partial ground', in giving our positive account of relative fundamentality. This way, we build an account with the desired scope.

As a start, the following necessary condition for relative fundamentality is suggested by discussion from the previous sections.

$(f$-NEC) $\forall x \forall y(f(y, x) \supset \neg n(x, y))$.

(For every $x$, for every $y$, if $y$ is more fundamental than $x$, then they are not alike in nature.)

So, for instance, although $\{\{$ Socrates $\}\}$ depends on $\{$ Socrates $\}$, given that $n(\{\{$ Socrates $\}\},\{$ Socrates $\})$, it follows from $(f$-NEC) by modus tollens that $\neg f(\{$ Socrates $\},\{\{$ Socrates $\}\})$. There is dependence without relative fundamentality. 
But we desire comparisons of relative fundamentality both between items which are not alike in nature and connected by dependence, but also between items that are not alike in nature but unconnected by dependence. This was revealed in the argument against (NEC). For instance, the account should predict that $f$ (Socrates, $\{$ Socrates $\}$ ) but also that $f$ (Plato, $\{$ Socrates $\}$ ).

We offer the following account of $f$, which includes both a 'direct' and 'indirect' disjunct, and where the indirect disjunct is itself disjunctive.

$$
\begin{aligned}
(f \text {-EQU) } & \forall x \forall y(f(y, x) \equiv(D \text { irect }) \neg n(x, y) \wedge \exists \phi(\phi(y) \wedge \phi<D x) \\
& \text { or }(\text { Indirect }) \text { (i) } \exists z(\neg n(x, y) \wedge n(z, y) \wedge \exists \phi(\phi(z) \wedge \phi<D x)) \\
& \text { or } \\
& \text { (ii) } \exists z(\neg n(z, y) \wedge n(x, z) \wedge \exists \phi(\phi(y) \wedge \phi<D z)) .{ }^{23}
\end{aligned}
$$

That is, $y$ is more fundamental than $x$ if and only if either (Direct) $x$ and $y$ are unalike in nature and $y$ is involved in some partial ground for $x$ 's being dependent, or (Indirect), which says that either (i) $y$ is alike in nature to something $z$, which is unalike in nature to $x$, and is involved in some partial ground for $x$ 's being dependent, or (ii) $x$ is alike in nature to something $z$, which is unalike in nature to $y$, and $y$ is involved some partial ground for $z$ 's being dependent. Let us consider some examples.

First, Socrates is more fundamental than $\{$ Socrates\}, since Socrates and \{Socrates\} are unalike in nature, and Socrates is a member of \{Socrates\} involves Socrates and partially grounds \{Socrates\} is dependent. This is an instance of (Direct). Furthermore, Socrates is more fundamental than the property being snub-nosed, since Socrates and being snub-nosed are unalike in nature, and Socrates instantiates being snub-nosed involves Socrates and partially grounds being snub-nosed is dependent. This is an instance of (Direct) involving generic dependence.

Second, Plato is more fundamental than $\{$ Socrates\}, since Socrates is alike in nature to Plato, but unalike in nature to $\{$ Socrates\}, and Socrates is a member of \{Socrates\} involves Socrates and partially grounds \{Socrates\} is dependent. This is an instance of (Indirect)(i). Furthermore, Plato is more fundamental than the property being snub-nosed, since Socrates is alike in nature to Plato, but unalike in nature to being snub-nosed, and Socrates instantiates being snub-nosed involves Socrates and partially grounds being snub-nosed is dependent. This is an instance of (Indirect) (i) involving generic dependence.

\footnotetext{
${ }^{23}$ We can say that something is absolutely fundamental, then, if there is nothing more fundamental than it. This allows for some dependence within the realm of the fundamental, assuming likeness in nature among the fundamental items.
} 
Third, Plato is more fundamental than the null set, since the null set is alike in nature to $\{$ Plato\}, but not alike in nature to Plato, and Plato is a member of $\{$ Plato $\}$ involves Plato and partially grounds $\{$ Plato $\}$ is dependent. This is an instance of (Indirect)(ii). Furthermore, Socrates is more fundamental than the property being a concrete abstractum, since being a concrete abstractum is alike in nature to being snub-nosed, but unalike in nature to Socrates, and Socrates instantiates being snub-nosed involves Socrates and partially grounds being snub-nosed is dependent. This, again, is an instance of (Indirect)(ii), but one involving an item which is not itself dependent. ${ }^{24}$ But the argument against (NEC) established that dependence is not necessary for relative fundamentality.

By extension of $f$, we might introduce a relation $f_{=}$, which holds between $x$ and $y$ if and only if $y$ is at least as fundamental if not more fundamental than $x$. So $f=$ is an extension of $f$, in the sense that $f$ is set theoretically included in $f_{=}$. Any two items are then $f_{=}$comparable: either $x$ is at least as fundamental if not more fundamental than $y$, or else $y$ is at least as fundamental if not more fundamental than $x$. That is, $f_{=}$is a total relation. There is, then, an account of $\left(f_{=}-\mathrm{EQU}\right)$ as follows.

$$
\left(f_{=} \text {-EQU) } \forall x \forall y\left(f_{=}(y, x) \equiv f(y, x) \vee n(x, y)\right) .\right.
$$

So $f_{=}(y, x)$ if and only if either $y$ is more fundamental than $x$, or else $x$ and $y$ are alike in nature. So, for instance, $f_{=}$(Socrates, Socrates $)$, since Socrates is alike in nature to himself. Moreover, $f_{=}($Socrates, Plato $)$since both Socrates and Plato are by nature human. ${ }^{25}$

Kathrin Koslicki (2013) raises the worry that, given (EQU) (i.e. that relative fundamentality is equivalent to ontological dependence), and the assumption that absolute fundamentality is identified with independence, it follows, e.g., that the null set is absolutely fundamental. This is an odd result, she claims, since the null set belongs to the same taxonomic category as any other set. I agree. In response, Koslicki suggests that the proponent of such a view should consider that dependence is merely a necessary condition for fundamentality, and not a sufficient one. Again, I agree, but add that it is not a necessary condition either.

\footnotetext{
${ }^{24}$ Alternatively, we might take the property to be dependent on some object that encodes it, if we're liberal about properties but tempted by a global claim of dependence that properties depend on their 'instances' (broadly construed, to include both exemplification and encoding). See (Linsky and Zalta 1994).

${ }^{25}$ Suppose there are disjoint fundamental domains, which are dissimilar in nature, and on which different items, which are dissimilar in nature, ultimately depend. How are the disjoint fundamentals, and disjoint derivatives, comparable with respect to fundamentality? Perhaps comparability is not desirable between disjoint domains.
} 
The proponent of (EQU) has a response to Koslicki's worry, if they adopt the view that the null set depends on the zero input. They are then no longer committed to saying that the null set is fundamental, since there is something on which it depends.

In any case, our account delivers the goods. For given that the null set is alike in nature to any other set, it is at least as fundamental if not more fundamental than any other set, given $\left(f_{-}-\mathrm{EQU}\right)$. Moreover, anything more fundamental than any other set is more fundamental than the null set, given ( $f$-EQU). (See the above example involving Plato and the null set.)

One final issue presents itself. For take some arbitrary non-set derivative item $d$ and some non-set fundamental (or basic) item $b$, and their singletons $\{d\}$ and $\{b\}$, and compare the singletons with respect to fundamentality. Given $n(\{b\},\{d\}),(f$-NEC) implies that $\neg f(\{b\},\{d\})$. But, it seems, $\{b\}$ and $\{d\}$ differ with respect to fundamentality in at least the following respect. In the case of $\{d\}$, there exists a many-link chain of relative fundamentality connecting $\{d\}$ to the fundamental, whereas in the case of $\{b\}$ there is no such many-link chain. Given a general presumption that items 'further' from the fundamental level are more derivative, $f(\{b\},\{d\})$. Contradiction.

But we should deny that distance suffices for relative fundamentality. Although it may well be that if $y$ is more fundamental than $x$ then there exists a longer chain connecting $x$ to the fundamental than any chain connecting $y$ to the fundamental, the converse is not the case. There are items dissimilar in distance but equal with respect to fundamentality, because alike in nature.

The upshot of $(f$-EQU $)$ and $\left(f_{=}-\mathrm{EQU}\right)$ is that they are predictively more successful than (EQU), by allowing for the separation of dependence and fundamentality, and without ideological superfluity. For relative fundamentality is given in terms of ontological dependence and likeness in nature. Both ontological dependence $(>)$ and likeness in nature $(n)$ are given within the ideology of essence. ${ }^{26}$

\section{Fundamentality in Aristotle's Categories}

We close with a discussion of fundamentality in Aristotle's Categories, and some puzzles that arise therein. We restrict discussion to the category of substance, and to the fundamentality structure within the category of substance. Within the category of substance, there are individuals, such as Socrates and Seabiscuit, which Aristotle labels 'primary substances'; and there are species, such as human and horse, and genera, such as animal and plant, which Aris-

${ }^{26}$ The notion of grounding also plays a role in harmonizing rigid and generic dependence. But it may not be strictly required. In any case, independent support for the ideology of grounding can be found, e.g., in (Fine 2012). 
totle labels 'secondary substances'. Moreover, there is a relation of being said $o f$, which structures the category of substance, and in virtue of which a substance is primary or secondary; and, among the secondary substances, in virtue of which a secondary substance is a species or genus.

An item in the category of substance is primary if and only if it is not the case that there is something of which it is said. Moreover, an item in the category of substance is secondary if and only if there is something of which it is said. ${ }^{27}$

Then Socrates and Seabiscuit, and individuals generally, are primary substances, as there is nothing of which they are said; and human, horse, animal, and plant, and species and genera generally, are each secondary substances, as human is said of Socrates, horse of Seabiscuit, animal of both Socrates and Seabiscuit, and plant of the tree just outside the window.

An item in the category of substance is a species if and only if there is something of which it is said (i.e. it is secondary) and, for every item of which it is said, that item is not said of anything (i.e. it is said only of primary substances). Then human, for instance, is a species, since it is said of Socrates, Plato, ..., each of which is primary. Moreover, an item in the category of substance is a genus if and only if there is something of which it is said (i.e. it is secondary) and there is something such that it is said of that thing and that thing is itself said of some further thing (i.e. the item is said of some secondary substance). Then animal, for instance, is a genus, as it is said of human, which is itself said of something (e.g. Socrates). ${ }^{28}$

The relation being said of is then taken to induce a structure of relative fundamentality on items within the category of substance. For Aristotle makes the following two claims of relative fundamentality.

I Primary substances are more fundamental-more a substancethan secondary substances, in virtue of the fact that secondary substances are said of primary substances but primary substances are not said of secondary substances $(2 \mathrm{a} 35-2 \mathrm{~b} 7) .{ }^{29}$

\footnotetext{
${ }^{27}$ We ignore differentiae in Categories, which are said of items in the category of substance but are not themselves in the category of substance.

${ }^{28}$ This is a bit of an oversimplification, since 'species' and 'genus' are relative terms. So we understand 'species' in this context as what Aristotle would call an 'infima species', i.e., the most specific species to which any item falling under it belongs.

Also, if, e.g., human is said of itself, then we might instead say that something is a species if and only if every distinct item of which it is said is pimary, and that something is a genus if and only if it is said of some distinct secondary item.

${ }^{29}$ In fact, he uses the superlative, saying that primary substances are substances 'most of all' (2a13-2a14). But it stands to reason that they are more a substance than secondary substances.
} 
II Among secondary substances, species are more fundamental than genera, in virtue of (a) being closer to primary substances, in the sense of being more revealing of the essences of primary substances; and (b) genera are said of species but species are not said of genera (e.g. animal is said of human being but not vice versa) $(2 \mathrm{~b} 8-2 \mathrm{~b} 22)$.

But the particular relative fundamentality judgments appear to be inconsistent given the following set of general claims in Categories. (Note that (iv) is a paraphrase of the passage quoted at the outset of the paper.)

i For any $x, y$ : if $y$ is said of $x$, then $y$ is a name of $x$ and the definition of $y$ is said of $x$ (2a19-2a2o).

ii For any $x, y$ : if $y$ is said of $x$ then, for every $z$, if $z$ is said of $y$ then $z$ is said of $x$ (1b1o-1b11).

iii For any $x, y$ : if $x$ and $y$ share both name and definition, then what $x$ is is the same as what $y$ is.

iv For any $x, y$ : if what $x$ is is the same as what $y$ is, then $x$ and $y$ do not differ with respect to fundamentality (3b32-4a9).

We assume that whatever is said of something gives the what-it-is of the item of which it is said, i.e., that 'said of' predications indicate essential predication. Take as given, then, that human is said of Socrates; that animal is said of human; and that animal is defined by percipient living substance.

Given that human is said of Socrates, and that animal is said of human, it follows from (ii) that animal is said of Socrates. Given that animal is defined by percipent living substance, it follows from (i), and the claim that animal is said of Socrates, that percipent living substance is said of Socrates. Moreover, given that animal is said of human, and that animal is defined by percipient living substance, it follows from (i) that human is defined by percipient living substance. So Socrates and human share a name (i.e. animal) and a definition (i.e. percipient living substance). By (iii), therefore, what Socrates is is the same as what human is. From (iv) it follows that Socrates and human do not differ with respect to fundamentality. But (i) implies that Socrates is more fundamental than human, since Socrates is a primary substance and human is a secondary substance.

Given the account of relative fundamentality developed in Section 4, we can quickly diagnose the problem. For there are two notions involved in a claim of relative fundamentality: dependence and likeness in nature. The example of Socrates and human would be a direct case of relative fundamentality, and so it must be that human is dependent, partially in virtue of something involving Socrates (i.e. that human is said of Socrates), and that 
Socrates and human are not alike in nature. The dependence claim seems solid. So the issue is with the claim that Socrates and human are not alike in nature. Indeed, the argument points in this direction. So ( $f$-EQU) offers good diagnostics here.

Perhaps the puzzle admits the following solution. We might think that species and genera, as such, are not by nature (or otherwise), for instance, rational animals, or by nature (or otherwise), for instance, percipient living substances. Rather, they are by nature said of such items. It is, then, only in a 'derivative' sense that superordinate items are said of them. This is perhaps indicated by Aristotle, when he says that "animal is predicated of man and therefore also of the individual man; [but] were it predicated of none of the individual men it would not be predicated of man at all" (2a36-2a38). We might take this as an expression of some sort of 'derivativeness'. That is, we might say that things are said of specific and generic substances in virtue of being said of individual substances, and in this sense predicating things of secondary substances is 'derivative'. We might conclude from this, then, that, appearances aside, Socrates and human are not alike in nature.

Perhaps the relations which really obtain between specific and generic substances are relations of subordination and superordination, which are distinct from being said of (and its converse). Then we can deny altogether that generic substances are said of specific substances, and still preserve dependence with the relation of subordination. ${ }^{30}$

But this points us in the direction of another puzzle. For we might raise a similar worry for Aristotle's claim that specific substances are more fundamental than generic substances. In this case, however, things are more challenging. For given that secondary substances are by nature said of other items in the category of substance, it is harder to sustain the claim that specific substances are unalike in nature to generic substances. Indeed, this sort of worry has historical precedent. For instance, Dexippus, in his On Aristotle's Categories, raises, and responds to, just this sort of worry.

SELUCUS: But if no substance admits of the more and less, how is the species said to be more a substance than the genus?

DEXIPPUS: Because it is not insofar as they are substances that they are involved in the more and less, but in virtue of their greater or lesser remove from sensible substance (46, 18-21, trans. Dillon).

Selecus's question is perhaps misleading. For Aristotle is clear that what he

${ }^{30}$ In this case, we should revise our definitions of species and genera as follows. An item in the category of substance is a species if and only if there is something of which it is said and there is nothing subordinate to it. Then an item in the category of substance is a genus if and only if there is something of which it is said and there is something subordinate to it. 
does not mean is that "one substance is not more a substance than another" (3b32-3b33). So merely saying that specific substances are more a substance, or more fundamental than, generic substances, is not indicative of a problem. There is only a problem if specific and generic substances are alike in nature, since Aristotle's claim is that "any given substance is not called more, or less, that which it is" (3b34).

Dexippus's response is interesting. For there would appear to be many ways in which two substances could be 'involved in the more and less' that have not any fundamentality commitments at all. For instance, Socrates may be taller than Plato, and so Socrates and Plato are involved in the more and less in virtue of Socrates' being taller than Plato. But we should hardly think that Socrates is more fundamental than Plato in virtue of being taller than him. For it is only in virtue of certain respects, namely essential respects, that involvement in the more and less is problematic.

But then Dexippus' response may appear more harmful than helpful. Take it as given that what Dexippus has in mind by "greater or lesser remove from sensible substance" is that specific substances are more revealing of the essences of primary substances than are generic substances (i.e. (II)(a) from above). But then insofar as the what-it-is of secondary substances is that they reveal the essences of primary substances, by virtue of being said of primary substances, then the fact that specific substances are more revealing of the essences of primary substances than are generic substances would appear to be an essential respect in virtue of which secondary substances are involved in the more and less (i.e. a respect insofar as they are what they are, or "insofar as they are [secondary] substances"). But this would violate Aristotle's claim that items alike in nature do not differ with respect to fundamentality. Thus there would appear to be a tension between the following four claims.

1. Secondary substances are by nature revealers of the essences of primary substances (i.e. they are by nature said of primary substances).

2. Specific substances are more a substance than generic substances.

3. Specific substances better reveal the essences of primary substances than do generic substances.

4. No items alike in nature differ with respect to fundamentality.

We shall not attempt to solve the puzzle here. But it is interesting that our account of relative fundamentality allows some way of making sense of how the four claims might hang together. However, this involves a slightly different reading of (2), and it relies on a subtlety involving (3). 
On (2), we might think that this is in fact a case of dependence without relative fundamentality. That is, generic substances depend on specific substances in virtue of relations of subordination, but, given (1), they are alike in nature, and so neither is more fundamental than the other. Perhaps the expression 'more a substance than' is ambiguous in this way, between substances depending on other substances, and between substances being more fundamental than other substances.

On (3), we might maintain that specific substances better reveal the essences of primary substances than do generic substances, but without violating (4). We need only specify how we do not interpret this claim. For it is not taken to mean that specific substances are, if you like, 'more said of' primary substances than are generic substances. For being said of does not admit of degrees in this way, just as our essential operator is such that no truth is 'truer' in virtue of an item than any other truth in virtue of that item. We might then grant that specific substances better reveal the essences of primary substances than do generic substances, but deny that this makes specific substances more what they are than generic substances. Perhaps this is why primary substances are substances "most strictly, primarily, and most of all", for they are both independent and fundamental.

In any event, our purposes have been served if our theory of fundamentality has been useful in articulating these puzzles in Categories and identifying their source. Whether the solutions entertained would survive scrutiny is for another occasion.

\section{Acknowledgements}

Thanks to Kathrin Koslicki, Riin Sirkel, Tuomas Tahko, and an anonymous referee for helpful comments.

\section{Bibliography}

Ackrill, J. (1963). Aristotle's Categories and De Interpretatione: Translated with Notes and Glossary, Clarendon Press, Oxford.

Barnes, E. (2012). Emergence and fundamentality, Mind 121: 873-901.

Bennett, K. (2011a). By our bootstraps, Philosophical Perspectives 25: 27-41.

Bennett, K. (2011b). Construction area (no hard hat required), Philosophical Studies 154: 79-104.

Cameron, R. (2008). Turtles all the way down: Regress, priority and fundamentality in metaphysics, Philosophical Quarterly 58: 1-14. 
Corkum, P. (2008). Aristotle on ontological dependence, Phronesis 53: 6592.

Correia, F. (2006). Generic essence, objectual essence, and modality, Noûs 40: $753-767$.

Dillon, J. (1990). Dexippus: On Aristotles Categories, Duckworth.

Fine, K. (1995a). The logic of essence, Journal of Philosophical Logic 24: 241273.

Fine, K. (1995b). Ontological dependence, Proceedings of the Aristotelian Society 95: 269-290.

Fine, K. (2009). The question of ontology, in D. Chalmers, D. Manley and R. Wasserman (eds), Metametaphysics: New Essays on the Foundations of Ontology, Oxford University Press, pp. 157-177.

Fine, K. (2010). Towards a theory of part, Journal of Philosophy 107: 559-589.

Fine, K. (2012). A guide to ground, in F. Correia and B. Schnieder (eds), Metaphysical Grounding: Understanding the Structure of Reality, Cambridge University Press, pp. 37-8o.

Koslicki, K. (2012). Varieties of ontological dependence, in F. Correia and B. Schnieder (eds), Metaphysical Grounding: Understanding the Structure of Reality, Cambridge University Press, pp. 186-2013.

Koslicki, K. (2013). Ontological dependence: An opinionated survery, in M. Hoeltje, B. Schnieder and A. Steinberg (eds), Varieties of Dependence, Philosophia Verlag, Munich, pp. 31-64.

Koslicki, K. (forthcoming). The coarse-grainedness of grounding, Oxford Studies in Metaphysics.

Linsky, B. and Zalta, E. (1994). In defense of the simplest quantified modal logic, in J. Tomberlin (ed.), Philosophical Perspectives 8: Philosophy of Logic and Language, Atascadero, Ridgeview, pp. 431-458.

Porphyry (1992). On Aristotle's Categories, Cornell University Press. Translated by Strange, $S$.

Schaffer, J. (2009). On what grounds what, in D. Chalmers, D. Manley and R. Wasserman (eds), Metametaphysics: New Essays on the Foundations of Ontology, Oxford University Press, pp. 347-383.

Tahko, T. (2012). Contemporary Aristotelian Metaphysics, Cambridge University Press. 